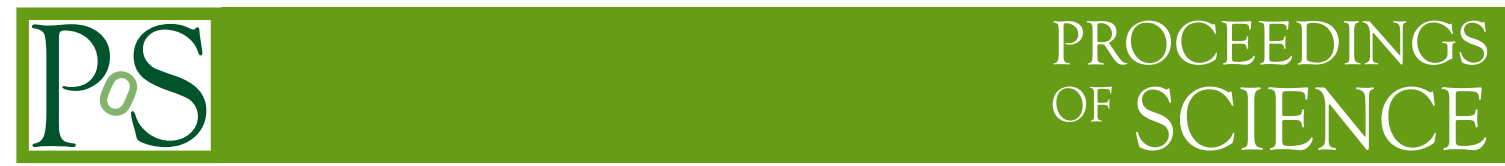

\title{
Magnetic versus baryonic jets for gamma-ray bursts
}

\section{Damien Bégué*}

Max-Planck Institut für extraterrestrische Physik

E-mail: dbegue@mpe.mpg. de

I discuss observed and theoretical constraints on the magnetization of gamma-ray burst (GRB) jets. Then, I describe a model based on the striped wind, and I explain how this model can help in solving the photon production problem in photospheric emission models of GRBs.

7th International Fermi Symposium

15-20 October 2017

Garmisch-Partenkirchen, Germany

\footnotetext{
* Speaker.
} 
The fireball model of gamma-ray bursts $[1,2,3]$ assumes that a large amount of energy is released in a compact region of typical size $r \sim 10^{7} \mathrm{~cm}$. The nature of the energy released is often assumed to be in the form of photons end electron-positron pairs. Yet, alternative scenarios assume that the energy released by the central engine of GRBs is mostly magnetic $[4,5,6]$. Once the energy is released, the jet is accelerated to relativistic speeds through magnetic or thermal pressure. Dissipation of energy if required to explain the non-thermal emission of GRBs. This can be achieved through magnetic reconnection [7, 8, 9] or shocks [3, 10, 11].

Observationally, clues on the magnetic content of GRB jets have been scrutinized for. As an example, it was argued that the absence of a black-body in the time-resolved spectra of GRB 080916C, one of the brightest GRB ever observed, is a clear smoking gun for a magnetized outflow [12]. Indeed, the more luminous the GRB, the more intense the black-body component should be. In the context of internal shocks scenarios, because of their low radiative efficiency, the lack of thermal component was explained by requiring that a large fraction of the total energy of the burst be magnetic [13]. The ICMART model [14] combines magnetized and thermal acceleration to explain the different observed spectra.

Not only the prompt phase was scrutinized, as additional constraints on the magnetization of jets can also be found in the afterglow. In fact, an x-ray plateau followed by a sharp decay ${ }^{1}$ has been frequently observed. It was argued that such a decrease in the observed flux could be attributed to the collapse of a central engine, like a pulsar, which was powering the emission of the plateau with its spin-down luminosity [15, 16, 17].

It has been proposed that GRBs central engine could be highly magnetized compact objects such as neutron star or black-hole [7, 18]. In such case, a striped wind can be created if the rotational axis and the dipole moment of the central object are not aligned. Acceleration to high Lorentz factor is powered by reconnection of the magnetic field. A schematic representation of the striped wind at large distances from the compact object is provided on Figure 1. It consists of regions of alternating magnetic polarity, separated by current sheets. If the striped wind has been extensively studied in the context of pulsar [19,20], the effects of radiation have been neglected, but they are expected to be important in the context of GRBs. A detailed derivation of the dynamics can be found in [21], in which the effect of radiation on the striped wind are studied. Here, we present only the results.

The effects of radiation are most apparent in the pressure balance condition of the current sheet

$$
n_{\mathrm{cs}} k_{\mathrm{B}} T_{\mathrm{cs}}+\frac{a_{\mathrm{th}} T_{\mathrm{cs}}^{4}}{3}=n_{\mathrm{m}} k_{\mathrm{B}} T_{\mathrm{m}}+\frac{a_{\mathrm{th}} T_{\mathrm{m}}^{4}}{3}+\frac{B^{2}}{8 \pi}
$$

where $n$ is the number density, $k_{\mathrm{B}}$ is the Boltzmann constant, $a_{\mathrm{th}}$ is the radiation constant and $B$ is the magnetic field strength. In addition, subscript cs and $\mathrm{m}$ represent the current sheet and the magnetized region respectively. It is also assumed that the radiation is in thermal equilibrium.

Two scenarios are found relevant in the context of GRBs. If radiation (mainly produced in the current sheet) cannot diffuse in the magnetized region, and remains in the current sheet, then $T_{\mathrm{cs}} \gg T_{\mathrm{m}}$. In this case $\mathrm{I}$, the pressure balance is satisfied by the radiation pressure. However, if diffusion of photon through the current sheet is efficient, then $T_{\mathrm{cs}} \simeq T_{\mathrm{m}}$ and the pressure balance condition is satisfied by the gas pressure, requiring $n_{\mathrm{cs}} \gg n_{\mathrm{m}}$ (case II). Figure 2 illustrates the

\footnotetext{
${ }^{1}$ With power-law temporal index larger than 3 .
} 


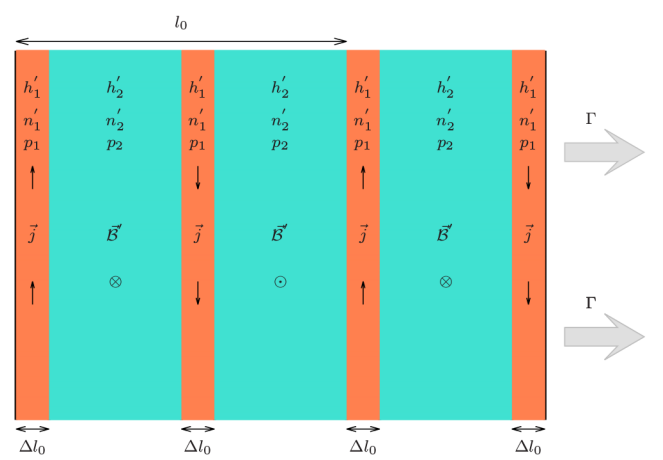

Figure 1: Details of the small length-scale structure of the striped wind: magnetized regions (in blue) of alternating polarity are separated by dense current sheets (in red). Both the pressure $p$ and the entropy $h$ have contributions from the gas and from the radiation: $p_{i}=p_{i, \mathrm{~g}}+p_{i, \text { rad }}$ and $h_{i}^{\prime}=h_{i, \mathrm{~g}}^{\prime}+h_{i, \mathrm{rad}}^{\prime}$. In addition, $\vec{j}$ and $\vec{B}$ are the current and the magnetic field. The outflow is moving at relativistic speed with Lorentz factor $\Gamma$. Finally, $\Delta$ is the relative width of the current sheet and $l_{0}$ is the wavelength of the striped wind.

difference between case I and case II. If the radial evolution of the Lorentz factor and temperature are similar in both cases, the ratio of densities $n_{\mathrm{cs}} / n_{\mathrm{m}}$ and the width $\Delta$ of the current sheet are very dissimilar.

In the context of GRBs, the problem is fully parametrized as function of the isotropic energy $L$, the maximum Lorentz factor $\Gamma_{\max }$, the size of one period of the striped wind $l_{0}$ and the product between the rate of magnetic reconnection $\varepsilon$ and the rotation period of the magnetized compact object $\Omega$, leading to the parameter $(\varepsilon \Omega)$. The radius at which the outflow becomes optically thin, which is the limit up to which our model is valid is given by

$$
r_{\mathrm{ph}}=\left\{\begin{array}{l}
6.8 \times 10^{11} L_{52}^{\frac{3}{5}}(\varepsilon \Omega)_{3}^{-\frac{2}{5}} \Gamma_{\max , 3}^{-1} \mathrm{~cm} \text { case I, } \\
7.0 \times 10^{11} L_{52}^{\frac{3}{5}}(\varepsilon \Omega)_{3}^{-\frac{2}{5}} \Gamma_{\max , 3}^{-1} \mathrm{~cm} \text { case II. }
\end{array}\right.
$$

The radius at which diffusion through the current sheet becomes efficient for case I is given by

$$
r_{\mathrm{D}, \mathrm{I}}^{\Delta}=6.05 \times 10^{10} L_{52}^{\frac{3}{5}}(\varepsilon \Omega)_{3}^{\frac{4}{5}} l_{0,7}^{\frac{6}{5}} \Gamma_{\max , 3}^{-1} \mathrm{~cm} .
$$

In the context of GRBs, the expansion of the outflow is described by case I, up to $r_{\mathrm{D}, \mathrm{I}}^{\Delta}$. Above this radius, photon pressure in the current sheet drops because of diffusion. The current sheet shrinks, its density increases and the transition is made to Case II.

Thanks to the large density in the current sheet, photon production through the Bremsstrahlung process is found to be efficient up to the radius

$$
r_{\mathrm{ff}, \mathrm{II}}^{\Delta}=2.2 \times 10^{11} L_{52}^{\frac{5}{9}}(\varepsilon \Omega)_{3}^{\frac{1}{9}} l_{0,7}^{\frac{16}{27}} \Gamma_{\max , 3}^{-\frac{20}{27}} \mathrm{~cm} .
$$

This radius is slightly smaller than the photospheric radius $r_{\mathrm{ph}}$. As such, photon production remains efficient almost up to the transparency. This could be a possible way to solve the photon starvation problem in the context of photospheric emission of GRBs [22, 23].

To conclude, I explain that several observations and theoretical arguments require the jet of (some) GRBs to be magnetized. Then, I discussed a model of magnetized outflow, based on the 

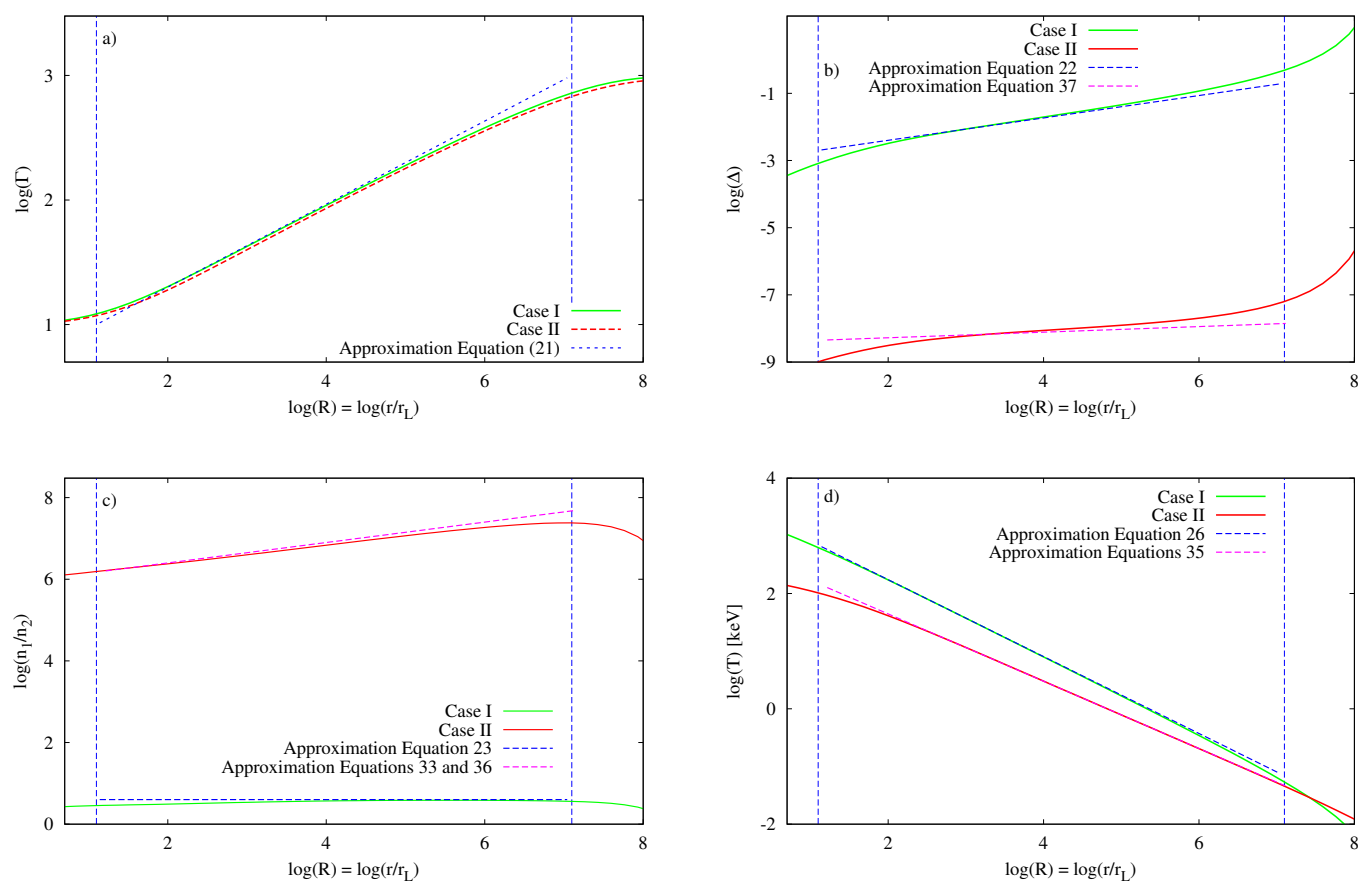

Figure 2: From top left to bottom right, radial evolution of the Lorentz factor $\Gamma$, of the current sheet width $\Delta$, of the ratio of densities $n_{\mathrm{cs}} / n_{\mathrm{m}}$, and of the temperature $T$. The blue and purple dashed lines represent the approximations derived in [21], with the corresponding equation number. Finally, the vertical blue dashed lines represent the beginning and end of the acceleration. Here $R=r / r_{\mathrm{L}}$ is the normalized radius, where $r_{\mathrm{L}}$ is the radius of the light-cylinder.

striped wind. It is produced by the rotation of a magnetized compact object. Particular attention was paid to the effects of radiation on the structure of the wind. I also discussed photon production mechanisms in this context, showing that Bremsstrahlung remains efficient almost up to the photosphere, which could provide a solution to the photon starvation problem in photospheric emission model for GRBs. The full derivation of the results can be found in [21].

\section{References}

[1] B. Paczynski, Gamma-ray bursters at cosmological distances, Astrophys. J. 308 (Sept., 1986) L43-L46.

[2] B. Paczynski, Super-Eddington winds from neutron stars, Astrophys. J. 363 (Nov., 1990) 218-226.

[3] M. J. Rees and P. Meszaros, Relativistic fireballs - Energy conversion and time-scales, Mon. Not. R. Astron. Soc. 258 (Sept., 1992) 41P-43P.

[4] R. D. Blandford and R. L. Znajek, Electromagnetic extraction of energy from Kerr black holes, Mon. Not. R. Astron. Soc. 179 (May, 1977) 433-456.

[5] M. Lyutikov, The electromagnetic model of gamma-ray bursts, New Journal of Physics 8 (July, 2006) 119, [astro-ph/0512342]. 
[6] A. Tchekhovskoy, J. C. McKinney and R. Narayan, Simulations of ultrarelativistic magnetodynamic jets from gamma-ray burst engines, Mon. Not. R. Astron. Soc. 388 (Aug., 2008) 551-572, [0803.3807].

[7] V. V. Usov, Millisecond pulsars with extremely strong magnetic fields as a cosmological source of gamma-ray bursts, Nature 357 (June, 1992) 472-474.

[8] C. Thompson, A Model of Gamma-Ray Bursts, Mon. Not. R. Astron. Soc. 270 (Oct., 1994) 480.

[9] G. Drenkhahn and H. C. Spruit, Efficient acceleration and radiation in Poynting flux powered GRB outflows, Astron. Astrophys. 391 (Sept., 2002) 1141-1153, [a stro-ph/0202387].

[10] M. J. Rees and P. Meszaros, Unsteady outflow models for cosmological gamma-ray bursts, Astrophys. J. 430 (Aug., 1994) L93-L96, [astro-ph/9404038].

[11] F. Daigne and R. Mochkovitch, Gamma-ray bursts from internal shocks in a relativistic wind: temporal and spectral properties, Mon. Not. R. Astron. Soc. 296 (May, 1998) 275-286, [astro-ph/9801245].

[12] B. Zhang and A. Pe'er, Evidence of an Initially Magnetically Dominated Outflow in GRB 080916C, Astrophys. J. 700 (Aug., 2009) L65-L68, [0 904 . 2943].

[13] R. Hascoët, F. Daigne and R. Mochkovitch, Prompt thermal emission in gamma-ray bursts, Astron. Astrophys. 551 (Mar., 2013) A124, [1302.0235].

[14] B. Zhang and H. Yan, The Internal-collision-induced Magnetic Reconnection and Turbulence (ICMART) Model of Gamma-ray Bursts, Astrophys. J. 726 (Jan., 2011) 90, [1 011.1197 ].

[15] E. Troja, G. Cusumano, P. T. O’Brien, B. Zhang, B. Sbarufatti, V. Mangano et al., Swift Observations of GRB 070110: An Extraordinary X-Ray Afterglow Powered by the Central Engine, Astrophys. J. 665 (Aug., 2007) 599-607, [astro-ph/0702220].

[16] H.-J. Lü and B. Zhang, A Test of the Millisecond Magnetar Central Engine Model of Gamma-Ray Bursts with Swift Data, Astrophys. J. 785 (Apr., 2014) 74, [1401.1562].

[17] H.-J. Lü, B. Zhang, W.-H. Lei, Y. Li and P. D. Lasky, The Millisecond Magnetar Central Engine in Short GRBs, Astrophys. J. 805 (June, 2015) 89, [1501. 02589].

[18] H. C. Spruit, F. Daigne and G. Drenkhahn, Large scale magnetic fields and their dissipation in GRB fireballs, Astron. Astrophys. 369 (Apr., 2001) 694-705, [astro-ph/ 0004274 ].

[19] F. V. Coroniti, Magnetically striped relativistic magnetohydrodynamic winds - The Crab Nebula revisited, Astrophys. J. 349 (Feb., 1990) 538-545.

[20] Y. Lyubarsky and J. G. Kirk, Reconnection in a Striped Pulsar Wind, Astrophys. J. 547 (Jan., 2001) 437-448, [astro-ph/0009270].

[21] D. Bégué, A. Pe'er and Y. Lyubarsky, Radiative striped wind model for gamma-ray bursts, Mon. Not. R. Astron. Soc. 467 (May, 2017) 2594-2611, [1610.03673].

[22] A. M. Beloborodov, Regulation of the Spectral Peak in Gamma-Ray Bursts, Astrophys. J. 764 (Feb., 2013) 157, [1207.2707].

[23] I. Vurm, Y. Lyubarsky and T. Piran, On Thermalization in Gamma-Ray Burst Jets and the Peak Energies of Photospheric Spectra, Astrophys. J. 764 (Feb., 2013) 143, [1209. 0763]. 